\title{
Determinants of treatment-related paradoxical reactions during anti- tuberculosis therapy: a case control study
}

Colin Stewart Brown ${ }^{1,2}$, Colette Joanne Smith³, Ronan Angus MacCormick Breen ${ }^{4}$, Lawrence Peter Ormerod ${ }^{5}$, Rahul Mittal ${ }^{5}$, Marie Fisk ${ }^{6}$, Heather June Milburn ${ }^{4}$, Nicholas Martin Price ${ }^{4}$, Graham Henry Bothamley ${ }^{7}$ and Marc Caeroos Isaac Lipman ${ }^{6,8^{*}}$ (D)

\begin{abstract}
Background: Inflammatory response following initial improvement with anti-tuberculosis (TB) treatment has been termed a paradoxical reaction (PR). HIV co-infection is a recognised risk, yet little is known about other predictors of $\mathrm{PR}$, although some biochemical markers have appeared predictive. We report our findings in an ethnically diverse population of HIV-infected and uninfected adults.

Methods: Prospective and retrospective clinical and laboratory data were collected on TB patients seen between January 1999-December 2008 at four UK centres selected to represent a wide ethnic and socio-economic mix of TB patients. Data on ethnicity and HIV status were obtained for all individuals. The associations between other potential risk factors and PR were assessed in a nested case-control study. All PR cases were matched two-to-one to controls by calendar time and centre.
\end{abstract}

Results: Of 1817 TB patients, 82 (4.5\%, $95 \%$ Cl 3.6-5.5 \%) were identified as having a PR event. The frequency of PR was $14.4 \%$ (18/125; $95 \%$ Cl 8.2-20.6 \%) and $3.8 \%$ (64/1692; 2.9-4.7) for HIV-positive and HIV-negative individuals respectively. There were no differences observed in PR frequency according to ethnicity, although the site was more likely to be pulmonary in those of black and white ethnicity, and lymph node disease in those of Asian ethnicity. In multivariate analysis of the case-control cohort, HIV-positive patients had five times the odds of developing PR $(\mathrm{aOR}=5.05 ; 95 \% \mathrm{Cl} 1.28-19.85, p=0.028)$, whilst other immunosuppression e.g. diabetes, significantly reduced the odds of PR ( $a \mathrm{OR}=0.01 ; 0.00-0.27, p=0.002$ ). Patients with positive TB culture had higher odds of developing PR ( $\mathrm{aOR}=6.87 ; 1.31-36.04, p=0.045)$ compared to those with a negative culture or those in whom no material was sent for culture. Peripheral lymph node disease increased the odds of a PR over 60-fold 4(9.60-431.25, $p<0.001)$.

Conclusion: HIV was strongly associated with PR. The increased potential for PR in people with culture positive TB suggests that host mycobacterial burden might be relevant. The increased risk with TB lymphadenitis may in part arise from the visibility of clinical signs at this site. Non-HIV immunosuppression may have a protective effect. This study highlights the difficulties in predicting PR using routinely available demographic details, clinical symptoms or biochemical markers.

\footnotetext{
* Correspondence: marclipman@nhs.net

${ }^{6}$ Royal Free London NHS Foundation Trust, Pond Street, London NW3 2QG, UK

${ }^{8}$ UCL Respiratory, Division of Medicine, University College London, Rowland Hill Street, London NW3 2PF, UK

Full list of author information is available at the end of the article
} 
(Continued from previous page)

Keywords: Tuberculosis, Treatment, Paradoxical reactions, HIV, IRIS, Predictors, Determinants, Ethnicity

Abbreviations: AFB, Acid Fast Bacilli; ART, Anti-retroviral therapy; CRP, C-reactive protein; ESR, Erythrocyte sedimentation rate; HIV, Human immunodeficiency virus; IQR, Inter-quartile range; IRIS, Immune Reconstitution Inflammatory Syndrome; MTB, Mycobacterium tuberculosis; NAAT, Nucleic Acid Amplification Technique; PR, Paradoxical reaction; TB, Tuberculosis

\section{Background}

Inflammatory response associated with treatment for tuberculosis (TB) has been termed a paradoxical reaction. This is defined as a worsening of clinical or radiological findings following the initiation of appropriate antituberculosis treatment, in the absence of evidence of disease relapse or the presence of another diagnosis [1]. HIV co-infection is a well-recognised risk factor for this, usually when concomitant anti-retroviral therapy (ART) is started [2] and is termed the Immune Reconstitution Inflammatory Syndrome (IRIS). This is thought to occur when a functioning immune response returns by the action of ART and worsens TB symptoms during treatment [3], or where undiagnosed TB is 'unmasked' by ART [4]. TB itself is immunomodulatory, and initiation of $\mathrm{TB}$ treatment can also reverse immunedysregulation [5].

In non-HIV positive people, the reported frequency of PR varies widely, but is lower than that seen in HIV positive people $[1,3]$. Further, there is some evidence that the immune response differs by ethnic group [6]. Some have considered that PR may occur as a result of pre-existing allergies to medication [7]. Despite advances in our understanding of the immunological profile of patients who develop IRIS, such as recognition of the role of tumour necrosis factor $\alpha$ (TNF- $\alpha$ ) and interleukin-6 [8] and other CD4 T-cell activation inflammatory cytokines and chemokines [9], and apart from introduction of ART in HIV-positive individuals, little is known regarding simple to measure baseline predictive factors for PR during anti-tuberculosis therapy [10]. There have been several, small case series from East Asia that have identified risk factors for developing PR. One Taiwanese study of 16 PR cases showed that decreased serum haemoglobin, albumin level, and lymphocyte count were associated with increased risk of pulmonary TB PR compared to over 600 patients who did not develop PR, although in multivariate analysis the differences just reached statistical significance at $5 \%$ [11]. Another study of 16 individuals from Hong Kong found that lower lymphocyte counts at baseline and extrapulmonary disease were identified risk factors compared to 53 patients who did not develop PR [12]. For pleural TB, younger age, high serum albumin level, low proportion of lymphocytes and high proportion of polymorphonuclear cells in pleural fluid was found in 32 patients in South Korea [13]. In another Korean study, 72 patients with pleural TB developed PR, with increased eosinophil count and protein levels in baseline pleural fluid identified as risk factors [14]. Earlier analysis from our group suggested that culture-positive TB predicted PR [15].

To investigate further associations, we conducted a multi-site UK study to determine the relationship between baseline presenting factors, ethnicity and reported PR frequency. Unlike previous studies, here we present patients with a wide range of ethnicities, and explore the impact of the site of active TB disease on PR, and the effect of HIV and the use of ART compared to other possible causes of PR.

\section{Methods}

\section{Study design}

Cohort study and nested case control study.

\section{Setting}

Four UK centres who were a collaborative group of TB partners with a wide mix of ethnicities across their patient populations. Anonymised demographic and clinical data were collated from the Homerton $(\mathrm{HH})$, Royal Free (RFH), and Guy's and St Thomas' Hospitals (STH) in London, and Royal Blackburn Hospital (RBH), Blackburn.

\section{Participants}

The cohort comprised all patients with a diagnosis of TB (culture-positive, or culture-negative with a response to TB treatment and no other possible diagnosis) recorded between January 2002 and December 2008 for RFH and HH, January 1999 and December 2008 (Blackburn) and mid-2006 and 2008 (STH, for all patients with electronic notes available). All data were retrospectively collated except for RFH, where half was prospectively collected as part of a study into PR. Baseline data for HIV status and collated ethnicities were recorded for all subjects based on United Kingdom Government's Office for National Statistics definitions [16]. For example, this grouping includes Black-British, Black-African and Black-Caribbean. PR was diagnosed using the clinical definition detailed above and defined by Breen et al [1]. Possible PR cases were then confirmed by the site specific leads and the principal author on review of patient notes. Two site leads maintained 
a prospective register of all consecutive $\mathrm{TB}$ cases $(\mathrm{HH}$ and $\mathrm{RBH}$ ), including those who has been identified as having a PR event. Two sites reviewed all case notes for evidence of PR (RFH and STH). Patients were followed up for the duration of treatment and reviewed according to local policy, normally one clinic visit after treatment cessation.

Those who defaulted follow-up or were found to have another diagnosis were not included in the final assessment. Sequential registration of patients was two to one matched to cases to assess risk factors for PR development, with those who definitely did not have any worsening of symptoms matched to those with a recorded PR. If there were any doubt regarding allocation or uncertainty of symptoms for controls then the subsequent registration was used to ensure that only definitive cases and controls were included in analysis. Figure 1 displays the participant groups.

\section{Variables}

Recorded information included basic demographic details; medical, drug, and allergy history; symptoms at TB diagnosis; physical examination; pre-treatment biochemical, haematological and inflammatory markers; radiographic and histological tests; TB diagnostic tests; and symptoms and evidence of PR.

\section{Statistical methods}

The overall incidence of PR at the four participating centres was calculated, with corresponding $95 \%$ confidence interval (CI). The incidence of PR according to HIV status and ethnic group was also calculated, and compared using a chi-squared test. Finally, the site of disease was summarised according to ethnicity to gain a descriptive understanding of any potential differences.

In the nested case-control study, potential factors associated with PR were examined. Parameters were chosen in advance of analysis: these were demographic factors (age, sex, and ethnicity); immunosuppression (HIV, non-HIV immunosuppression, and the potential immune-modulatory lifestyle factors tobacco and alcohol use); baseline TB disease site, symptoms \& microbiology; and baseline blood parameters (total white cell count, lymphocyte count, haemoglobin; albumin and alkaline phosphatase; C-reactive protein and erythrocyte sedimentation rate; lactose dehydrogenase and vitamin D levels).

Analyses were performed using conditional logistic regression to account for matching by calendar time and centre. In order to arrive at a parsimonious model, those potential factors with $p \leq 0.1$ in univariate analyses were included in the multivariate model, along with a priori assumptions that were brought forward for inclusion (age and sex as routine, and HIV status and TB culture result from our previous findings). No formal adjustments for multiple comparisons were made. Missing data were accounted for with categorical variables, although missing baseline blood tests were not imputed, and analysis limited to those with a blood test available. STROBE guidelines were adhered to for reporting [17].

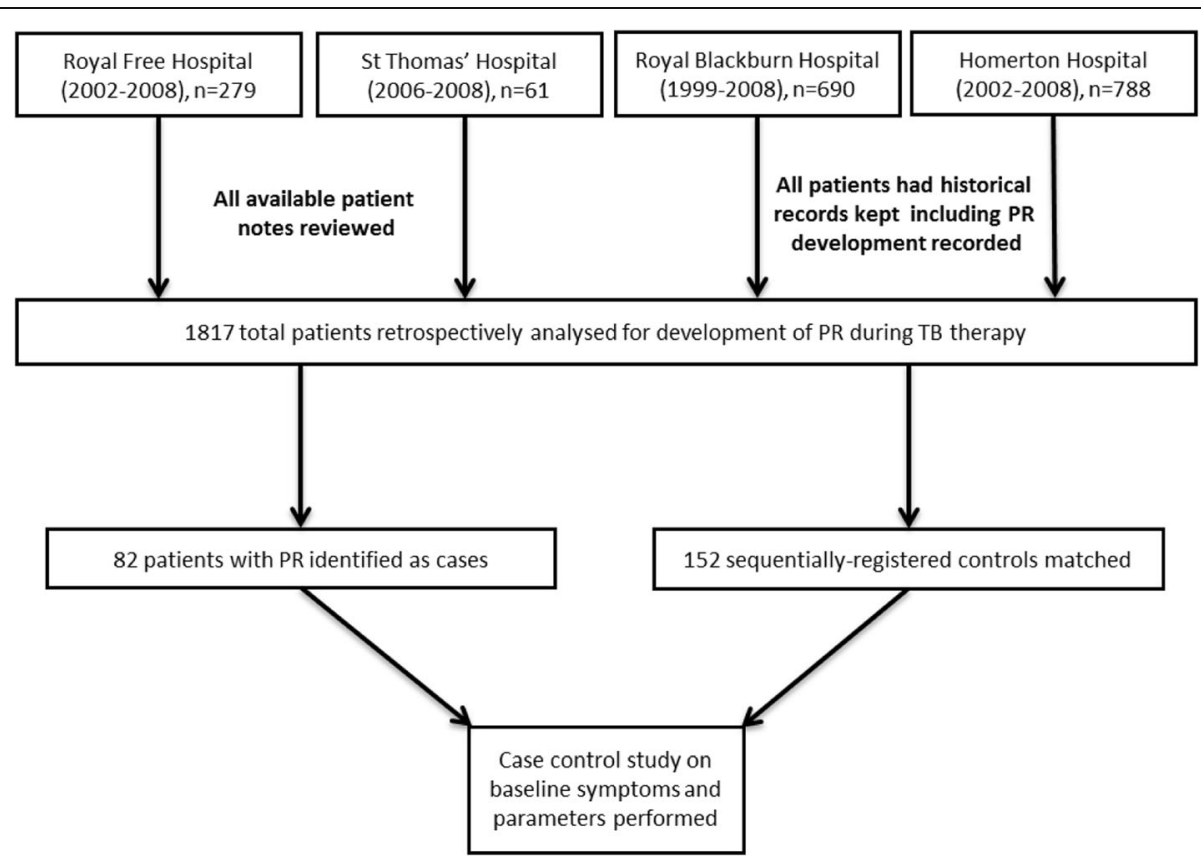

Fig. 1 Study population 


\section{Results}

\section{Total patient group}

Of 1817 patients treated for TB for whom records were reviewed, 82 (4.5 \%, 95 \% CI 3.6-5.5 \%) subjects with PR were identified. Fifty-five percent of PR cases were female, with a median age of 32 years (IQR 26-41). Twenty-two percent of people with PRs were HIV coinfected (compared to an overall prevalence of $6.9 \%$ of the total 1817 patients). The frequency of PR in the HIV positive group was $14.4 \%$ (95\% CI 8.2-20.6\%) compared to $3.8 \%$ (95\% CI 2.9-4.7) in the HIV negative or untested group $(p<0.001)$.

Table 1 shows the proportion of PR within different racial groups, disaggregated by HIV status. There was no difference in frequency of PR among the different ethnicities in either the HIV positive or HIV negative or unknown groups $(p=0.73$ and $p=0.15)$. Information on the proportion of patients tested for HIV was available for $\mathrm{HH}$ (where testing was performed in $100 \%$ of cases), $\mathrm{RFH}$ (testing in $95 \%$ ) and STH (92\% cases). At RBH, this was not routinely performed however all patients from injecting drug users or sub-Saharan Africa were tested.

Amongst the 82 individuals who experienced PR, the site of disease varied by ethnic group (Table 2). Extrapulmonary lymph node involvement was found to be significantly more common among South Asians than Blacks adjusted for other ethnicities $(p=<0.001)$. Those of Black ethnicity conversely exhibited more pulmonary involvement than people of South Asian and White ethnicity $(p=0.045)$. There was a higher prevalence of systemic symptoms such as fever and sweating amongst those of black and white, compared to South and East Asian ethnicity (40 and $50 \%$ versus 2 and $17 \%$ respectively; $p=0.004)$. HIV co-infection was more common in these PR populations ( $47 \%$ in the Black PR population and $38 \%$ in the White, none in other groups), though HIV status was not measured in 18 (22\%) of cases. Of note, within the HIV negative population, there was no greater prevalence of baseline systemic symptoms across ethnicities compared to the HIV positive population $(p=0.485)$. Where timing of ART initiation was recorded in HIV positive patients, ART was started prior to or at the time of PR development in 10 cases (56\%) and following TB treatment in 5 cases (28\%). 3 HIV positive patients were not recorded as receiving ART (17\%).

The majority of PR occurred at the presenting site of TB disease (81; $88 \%)$, with $91 \%$ (84) having an exacerbation of pre-diagnosis symptoms. The median time of onset of PR was at week 4.5 (IQR 3-11, maximum of 150 weeks in one patient who represented after treatment completion). The median duration of PR was 4 weeks (range 1-46 weeks). Forty-five percent (41) received treatment with steroids.

\section{Case control analysis}

In total 152 controls were selected for the 82 cases; 12 cases could only be matched to one control, and 70 cases were matched to two controls. Table 3 provides information on selected demographics, baseline inflammatory markers, allergies reported to medication, TB smear and culture, and presenting symptoms in the PR and non-PR groups. There were no observed differences in age or sex between cases or controls. Pulmonary disease was more common as the presenting site of initial disease in controls (63\% versus $38 \%$ ), and peripheral lymph nodes more frequent in PRs (42\% versus $3 \%$; $p<0.001)$. There were somewhat more HIV-positive individuals in the PR group (22\% versus $16 \%$; $p=0.41$ ).

A positive mycobacterial culture and Nucleic Acid Amplification Technique (NAAT), lack of tobacco and alcohol use, a raised baseline ESR, the absence of nonHIV immunosuppression (defined here as diabetes mellitus, cancer, chronic renal failure or pregnancy), and presence of initial site of disease at extrathoracic lymph nodes were associated with development of PR in univariate analysis. In multivariate analysis (Table 4), presence of baseline LN disease, TB culture positivity, HIV positive status, and presence of non-HIV immunosuppression were all associated with the development of PR at a $5 \%$ level of significance.

Table 1 Ethnicity, HIV status and PR

\begin{tabular}{|c|c|c|c|c|c|}
\hline \multirow[t]{2}{*}{ Ethnicity } & \multirow{2}{*}{$\begin{array}{l}\text { Total } \\
\text { number }\end{array}$} & \multirow{2}{*}{$\begin{array}{l}\text { Number HIV } \\
\text { positive (\%) }\end{array}$} & \multicolumn{3}{|c|}{ Paradoxical reaction number (\%; 95 \% confidence intervals) } \\
\hline & & & Total population & HIV positive & HIV negative/unknown \\
\hline Black & 555 & $96(17.3)$ & $32(5.8 ; 3.7-7.5)$ & $15(15.6 ; 8.4-22.9)$ & $17(3.7 ; 2.0-5.4)$ \\
\hline East Asian & 98 & $5(5.1)$ & $6(5.1 ; 1.4-10.9)$ & - & $6(6.5 ; 1.5-11.4)$ \\
\hline Mixed Race & 11 & - & 0 & - & - \\
\hline Other & 50 & $4(13.3)$ & $3(6.3 ; 0-12.6)$ & - & $3(6.5 ; 0.0-13.7)$ \\
\hline South Asian & 786 & $2(2.5)$ & $33(5.7 ; 2.8-5.6)$ & - & $33(4.2 ; 2.6-5.6)$ \\
\hline White & 317 & $18(5.7)$ & $8(5.1 ; 0.8-4.3)$ & $3(16.7 ; 0-33.9)$ & $5(1.7 ; 0.2-3.1)$ \\
\hline Total & 1817 & $125(6.9)$ & $82(4.5 ; 3.6-5.6)$ & $18(14.4 ; 8.2-20.6)$ & $64(3.8 ; 2.9-4.7)$ \\
\hline
\end{tabular}


Table 2 Site of PR by ethnicity

\begin{tabular}{|c|c|c|c|c|c|}
\hline \multirow[t]{2}{*}{ Site of $\mathrm{PR}^{\mathrm{a}}$} & \multicolumn{5}{|c|}{ Number of patients by ethnicity (percentage of total ethnic group with PR) } \\
\hline & Black & East Asian & Other & South Asian & White \\
\hline Total & $32(100 \%)$ & $6(100 \%)$ & $3(100 \%)$ & $33(100 \%)$ & $8(100 \%)$ \\
\hline Chest $^{\mathrm{b}}$ & $15(47 \%)$ & $1(17 \%)$ & - & $6(18 \%)$ & $1(13 \%)$ \\
\hline Systemic Symptoms ${ }^{c}$ & $13(40 \%)$ & $1(17 \%)$ & - & $2(6 \%)$ & $4(50 \%)$ \\
\hline Abdominal & $6(19 \%)$ & - & - & - & $1(13 \%)$ \\
\hline Brain & $2(6 \%)$ & - & - & - & - \\
\hline Peripheral lymph nodes & $8(25 \%)$ & $4(67 \%)$ & $3(100 \%)$ & $25(76 \%)$ & $4(50 \%)$ \\
\hline Other & $2(6 \%)$ & $1(17 \%)$ & 1 (33 \%) & $1(3 \%)$ & $3(38 \%)$ \\
\hline
\end{tabular}

${ }^{\mathrm{a} P R}$ may occur at more than one site for each patient, so percentages do not sum to $100 \%$

${ }^{b}$ Pulmonary, pleural, and mediastinal lymph nodes

'Systemic symptoms are persistent fever and persistent night sweats

The association with HIV was confirmed in multivariate analysis, where the odds ratio of developing PR were 5.05 (95\% CI 1.28-19.85, $p=0.028$ ) compared to those without known HIV infection.

Lymph node disease was associated with a 64.33-fold increased odds of having a PR event (95 \% CI 9.60$431.25, p<0.001)$. A positive TB culture increased the odds of developing PR 6.87 times (95\% CI 1.31-36.04, $p=0.045)$. Though NAAT showed an association, the greatest percentage difference in the non-PR and PR groups was in those who did not have a test performed. Finally, non-HIV immunosuppression reduced the risk of PR significantly (aOR 0.01; $95 \%$ CI $0.00-0.27$, $p=0.002$ ).

Baseline ESR could not be fitted within the multivariate model due to the limited numbers of people in whom this was measured ( $n=48$ in cases, $n=91$ in controls, only 97 of which were able to be used in matched case control analysis; see Table 3). Sensitivity analysis dividing ESR into either three or four categories confirmed increasing odds of PR with increasing ESR, but again this could not be fitted into multivariate analysis due to small numbers. ESR is often moderately elevated in HIV infection [18]. In univariate analysis a 1-log increase in ESR was associated with a 4.43 increased odds of developing PR (95 \% CI 1.30-15.1); this association remained in sensitivity analysis when adjusted for HIV status (aOR 4.78; $1.29-17.72, p=0.019$ ).

\section{Discussion}

Within our total population, we find that HIV is strongly associated with development of PR, mainly in the context of ART initiation within the case control cohort (55\% started ART before or at time of PR development). The observed frequency of $14.4 \%$ within the HIV positive group is similar to that of $15.7 \%$ reported in a meta-analysis of $3500 \mathrm{HIV}$ co-infected patients [19]. In the HIV negative or unknown group our observed frequency of $3.8 \%$ is also in line with published observations (ranging from $2 \%$ in all patients to $25 \%$ in extra-pulmonary tuberculosis) $[1,2,20]$. The long length of time to PR onset that can occur (up to a maximum of 150 weeks in our cohort) has been previously recognised [21], and our observed median time to onset is similar to other general studies [1], though shorter than those looking solely at extrapulmonary PR [20].

We observed an association between the location of the PR site and ethnicity, although the overall rate of PR did not differ between ethnic groups. This likely arises because baseline disease site (which does appear to have a racial origin) heavily influences the site of PR: our Black and White study populations had more pulmonary disease compared to those of South Asian ethnicity, in whom lymphadenitis predominates. This is in keeping with other reports [22].

Our findings suggest that peripheral lymph node disease greatly predisposes to PR; despite large confidence intervals, the increased risk is pronounced, in the magnitude of sixty-fold increased odds in multivariate analysis. This has been previously documented as a likely site of IRIS. In the third British Thoracic Society lymph node study, carried out in a predominantly South Asian cohort between 1987-89 i.e. largely without HIV co-infection, new lymphadenopathy was reported in $16 \%$ in the first three months of treatment [23], and new nodes or sinuses in $7-12 \%$ after treatment completion [24]. We cannot exclude ascertainment bias as a possible cause of this; and PR at other less easily-identifiable locations might be under-reported in the absence of systemic symptoms justifying further investigations. This potential confounding supports the need for an international consensus to ensure that all potential paradoxical reactions are appropriately identified and recorded to improve epidemiology and surveillance [25].

As reported with TB meningitis IRIS in South Africa [26] and disseminated TB in a Malaysian HIV cohort [27], the increased potential for PR in people with 
Table 3 Univariate analysis of baseline variables at TB diagnosis in case control cohort

\begin{tabular}{|c|c|c|c|c|}
\hline \multicolumn{2}{|l|}{ Variable ( $N=234$ total unless otherwise stated) } & $\mathrm{PR}(\%)(N=82)$ & Matched control without PR $(N=152)$ & $P$ value ${ }^{*}$ \\
\hline \multicolumn{5}{|l|}{ Demographics $^{a}$} \\
\hline \multicolumn{2}{|l|}{ Age (Mean) } & 33.7 & 37.0 & 0.162 \\
\hline \multirow[t]{2}{*}{ Sex (\%) } & Male & $72(45 \%)$ & $72(47 \%)$ & - \\
\hline & Female & $45(55 \%)$ & $80(53 \%)$ & 0.770 \\
\hline \multirow[t]{5}{*}{ Ethnicity (\%) } & White & $8(10 \%)$ & $26(17 \%)$ & - \\
\hline & Black & $32(39 \%)$ & $65(43 \%)$ & - \\
\hline & East Asian & $6(7 \%)$ & $9(6 \%)$ & - \\
\hline & South Asian & $33(40 \%)$ & $46(30 \%)$ & - \\
\hline & Other & $3(4 \%)$ & $6(4 \%)$ & 0.663 \\
\hline \multicolumn{5}{|l|}{ Baseline TB disease site, symptoms \& microbiology } \\
\hline \multirow[t]{5}{*}{ Site (\%) } & Chest $^{\mathrm{b}}$ & $29(35 \%)$ & $96(63 \%)$ & - \\
\hline & Abdominal & $2(2 \%)$ & $4(3 \%)$ & - \\
\hline & Brain & $2(2 \%)$ & $5(3 \%)$ & - \\
\hline & Peripheral lymph nodes & $34(41 \%)$ & $4(3 \%)$ & - \\
\hline & Other or mixed sites & $15(18 \%)$ & $43(28 \%)$ & $<0.001$ \\
\hline \multirow[t]{3}{*}{ Systemic Symptoms (\%) (with weight loss) } & Yes & $23(28 \%)$ & $45(30 \%)$ & - \\
\hline & No & $54(66 \%)$ & $101(66 \%)$ & - \\
\hline & Not recorded & $5(6 \%)$ & $6(4 \%)$ & 0.901 \\
\hline \multirow[t]{3}{*}{ Systemic Symptoms (\%) ${ }^{c}$ (without weight loss) } & Yes & $27(33 \%)$ & $64(42 \%)$ & - \\
\hline & No & $50(61 \%)$ & $82(54 \%)$ & - \\
\hline & Not recorded & $5(6 \%)$ & $6(4 \%)$ & 0.332 \\
\hline \multirow[t]{3}{*}{ Acid Fast Bacilli (\%) } & Negative & $47(57 \%)$ & $88(58 \%)$ & - \\
\hline & Positive & $25(30 \%)$ & $42(28 \%)$ & - \\
\hline & Not performed & $10(12 \%)$ & $22(14 \%)$ & 0.770 \\
\hline \multirow[t]{3}{*}{ M. tuberculosis NAAT (\%) } & Negative & $8(10 \%)$ & $9(6 \%)$ & - \\
\hline & Positive & $20(24 \%)$ & $24(16 \%)$ & - \\
\hline & Not performed & $54(66 \%)$ & 119 (78 \%) & 0.007 \\
\hline \multirow[t]{3}{*}{ M. tuberculosis Culture (\%) } & Negative & $13(16 \%)$ & $37(24 \%)$ & - \\
\hline & Positive & $62(76 \%)$ & $104(68 \%)$ & - \\
\hline & Not performed & 7 (9\%) & $11(7 \%)$ & 0.256 \\
\hline \multicolumn{5}{|l|}{ Immune system functioning } \\
\hline \multirow[t]{3}{*}{ HIV status (\%) } & Negative & $46(56 \%)$ & $101(66 \%)$ & - \\
\hline & Positive & $18(22 \%)$ & $25(16 \%)$ & - \\
\hline & Not recorded & $18(22 \%)$ & $26(17 \%)$ & 0.413 \\
\hline \multirow[t]{3}{*}{ Drug allergies (\%) } & No & $34(41 \%)$ & $60(39 \%)$ & - \\
\hline & Yes & $2(2 \%)$ & $2(1 \%)$ & - \\
\hline & Not recorded & $46(56 \%)$ & $90(59 \%)$ & 0.688 \\
\hline \multirow[t]{3}{*}{ Immunosuppression (\%) } & No & $80(98 \%)$ & $130(8 \wedge \%)$ & - \\
\hline & Yes & $1(1 \%)$ & $17(11 \%)$ & - \\
\hline & Not recorded & $1(1 \%)$ & $5(3 \%)$ & 0.004 \\
\hline \multicolumn{5}{|l|}{ Potential immune-modulating lifestyle factors } \\
\hline \multirow[t]{3}{*}{ Tobacco use (\%) } & No & $60(73 \%)$ & $97(64 \%)$ & - \\
\hline & Yes & $10(12 \%)$ & $37(24 \%)$ & - \\
\hline & Not recorded & $12(15 \%)$ & $18(12 \%)$ & 0.101 \\
\hline
\end{tabular}


Table 3 Univariate analysis of baseline variables at TB diagnosis in case control cohort (Continued)

\begin{tabular}{|c|c|c|c|c|}
\hline \multirow[t]{3}{*}{ Alcohol use (\%) } & No & $59(72 \%)$ & $85(56 \%)$ & - \\
\hline & Yes & $8(10 \%)$ & $34(22 \%)$ & - \\
\hline & Not recorded & $15(18 \%)$ & $33(22 \%)$ & 0.007 \\
\hline \multicolumn{5}{|c|}{ Geometric mean (range) baseline blood test results ${ }^{\mathrm{e}}$ (units, number of patients with variable data) } \\
\hline White blood cell count (cells/ $\mu \mathrm{L}, n=229$ ) & & $7.2(3.1-16.1)$ & $7.2(2.1-18.4)$ & 0.953 \\
\hline Lymphocyte Count (cells/ $\mu \mathrm{L}, n=229$ ) & & $1.6(0.4-4.2)$ & $1.6(0.3-6.5)$ & 0.855 \\
\hline Haemoglobin (g/dL, $n=230)$ & & $11.7(7.7-16.0)$ & $12.0(7.1-17.3)$ & 0.285 \\
\hline Albumin $(\mathrm{g} / \mathrm{dL}, n=225)$ & & $38(16-77)$ & $37(9-50)$ & 0.501 \\
\hline Alkaline Phosphatase $(U / L, n=225)$ & & $134(44-823)$ & $123(14-1074)$ & 0.627 \\
\hline C-Reactive Protein (mg/L, $n=203$ ) & & $62(1-280)$ & $65(1-396)$ & 0.939 \\
\hline Erythrocyte Sedimentation Rate (mm/h, $n=139)$ & & $56(6-132)$ & $46(2-129)$ & 0.017 \\
\hline Vitamin D Levels ( $\mathrm{ng} / \mathrm{mL}, n=85)$ & & $27.1(8.6-79.4)$ & $30.9(6.3-168.0)$ & 0.764 \\
\hline Lactate Dehydrogenase $(\mathrm{U} / \mathrm{L}, \mathrm{n}=99)$ & & $499(294-1156)$ & $523(246-2127)$ & 0.699 \\
\hline
\end{tabular}

${ }^{*} P$ values calculated using Wald tests in conditional logistic regression

a Demographic information available for all patients

${ }^{\mathrm{b}}$ Pulmonary, pleural, and mediastinal lymph nodes

'Systemic symptoms are persistent fever and persistent night sweats

${ }^{\mathrm{d}}$ Non-HIV immunosuppressive state such as diabetes mellitus, cancer, chronic renal failure or pregnancy

${ }^{\mathrm{e}}$ Log means were used for analysis to approximate normal distributions for skewed tails

culture positive tuberculosis confirms that underlying host mycobacterial burden appears important in the development of PR, though some culture negative disease may not in fact be TB but rather another diagnosis. Therefore culture positive disease could be over-represented artefactually in analysis. It should be noted, however, that we also found PRs in apparent low mycobacterial burden disease (as $16 \%$ were culture negative at baseline).

There was evidence that the combined group of non-HIV immunosuppression including diabetes was associated with reduced odds of developing PR. Whilst the numbers in this heterogeneous population are small $(n=18$, seven of whom were diabetic, and none were using or recently withdrawn from biologic treatments) with wide confidence intervals, PR has occurred in oncology patients receiving granulocyte colony-stimulating factor post chemotherapy, though not when functionally impaired [28], and on TNF- $\alpha$ antagonist withdrawal [29]. We are unaware of a reported association between PR and diabetes, and this subgroup should be explored in larger datasets.

Although numbers were small, we observed a trend towards a lower risk of developing PR with increased alcohol use (aOR 0.21; 0.04-1.01; group categorical $p$ value of 0.009 , alcohol use versus no alcohol, $p=0.051$ ). This, again, would be interesting to further evaluate in a larger study.

Recent studies have suggested that baseline C-Reactive Protein (CRP) is associated with the risk of developing PR in HIV co-infected individuals [30, 31]. This requires confirmation in a larger longitudinal study. From our data it appears that the Erythrocyte Sedimentation rate (ESR), which has also been shown to be raised at baseline in TB [32] may be a useful predictive marker or PR. Given the limited data on ESR within our cohort, this will require further work to determine whether an association exists.

\section{Strengths and limitations}

There are several limitations of this study. Ascertainment of development of PR was collected retrospectively at two centres, and therefore we cannot be certain all true PR cases were identified, although case notes were reviewed thoroughly by both the site PI and the study lead author. Furthermore, the case control nature of the primary analysis limits the potential biases caused by this. Selection bias may result in identification of the more severe cases that required treatment. In particular for some blood measures, not all tests were performed in all patients. For the total population, HIV testing was not performed in every patient and although testing was routinely offered at the diagnosis of $\mathrm{TB}$, there is the potential for HIV status to be assessed more assiduously in diagnosed or suspected PR cases. The one setting where this was not performed regularly has a low overall HIV prevalence. In our study, where known, overall testing rates for HIV were above $90 \%$. Given the relatively small numbers of PR, some confidence intervals are wide in multivariate analysis. However the large baseline denominator from whom the controls were drawn had a broad representation of age, ethnicity, sex, and baseline disease. We believe our findings highlight areas for future study, and can be generalised to other population settings. 
Table 4 Multivariate analysis of factors associated with development of PR: results from conditional logistic regression model

\begin{tabular}{|c|c|c|c|}
\hline \multicolumn{2}{|l|}{ Variable } & $\begin{array}{l}\text { Odds ratio } \\
(95 \% \mathrm{Cl})\end{array}$ & $P$ value \\
\hline \multicolumn{2}{|l|}{ Age } & $\begin{array}{l}0.98(0.95-1.02) \\
\text { per increasing } \\
\text { year }\end{array}$ & 0.409 \\
\hline \multicolumn{2}{|l|}{ Sex } & $\begin{array}{l}0.57(0.19-1.67) \\
\text { for females }\end{array}$ & 0.302 \\
\hline \multirow[t]{5}{*}{ Site } & Chest & 1.00 & - \\
\hline & Abdominal & $8.11(0.18-356.01)$ & - \\
\hline & Brain & $2.22(0.22-23.30)$ & - \\
\hline & $\begin{array}{l}\text { Peripheral lymph } \\
\text { nodes }\end{array}$ & $64.33(9.60-431.25)$ & - \\
\hline & Other or mixed sites & $1.23(0.41-3.74)$ & $<0.001$ \\
\hline \multirow[t]{3}{*}{ HIV Status } & Negative & 1.00 & - \\
\hline & Positive & $5.05(1.28-19.85)$ & - \\
\hline & Not recorded & $0.50(0.02-14.97)$ & 0.028 \\
\hline \multirow[t]{3}{*}{ Immunosuppression } & No & 1.00 & - \\
\hline & Yes & $0.01(0.00-0.27)$ & - \\
\hline & Not recorded & $0.13(0.00-0.90)$ & 0.002 \\
\hline \multirow[t]{3}{*}{ Tobacco use } & No & 1.00 & - \\
\hline & Yes & $0.71(0.17-2.85)$ & - \\
\hline & Not recorded & $3.36(0.39-21.04)$ & 0.462 \\
\hline \multirow[t]{3}{*}{ Alcohol use } & No & 1.00 & - \\
\hline & Yes & $0.21(0.04-1.01)$ & - \\
\hline & Not recorded & $0.01(0.01-0.56)$ & 0.009 \\
\hline $\mathrm{ESR}^{\mathrm{a}}$ & Mean (mm/h) & $\begin{array}{l}\text { Could not fit } \\
\text { within model }\end{array}$ & - \\
\hline \multirow[t]{6}{*}{ TB Diagnosis } & $\%$ NAAT negative & 1.00 & - \\
\hline & $\%$ NAAT positive & $1.23(0.11-12.63)$ & - \\
\hline & $\begin{array}{l}\% \text { NAAT not } \\
\text { performed }\end{array}$ & $0.10(0.01-1.11)$ & 0.009 \\
\hline & $\%$ culture negative & 1.00 & - \\
\hline & $\%$ culture positive & $6.87(1.31-36.04)$ & - \\
\hline & $\begin{array}{l}\% \text { culture not } \\
\text { performed }\end{array}$ & $3.81(0.66-22.14)$ & 0.045 \\
\hline
\end{tabular}

${ }^{a}$ ESR was only performed on a limited number of patients $(n=139$, only 97 of which could be used for case control analysis): it was unable to be included in the model due to small numbers. In univariate analysis a 1-log increase in ESR was associated with a 4.43 increased odds of developing PR (95 \% Cl 1.30-15.1); this increased to $4.78(1.29-17.72)$ when adjusted for HIV status

\section{Conclusions}

Overall, this retrospective case control study highlights the known association between HIV and PR during TB treatment. It did not succeed in identifying any clear additional positive or negative risk factors apart from possible mycobacterial burden and non-HIV immunosuppression; or confirm previous blood parameters from smaller studies. This demonstrates the difficulties in predicting PR using routinely available demographic details, clinical symptoms or biochemical markers. It also highlights the apparent frequency of PR when it occurs at visibly apparent sites such as peripheral lymph nodes. To determine whether PR risk stratification, based on easy to measure variables at initial presentation is possible, greater numbers of prospectively-gathered PR cases need to be assessed. A positive result would enable people at highest risk to be monitored carefully, with appropriate therapy started to treat, or even prevent PR.

\section{Acknowledgements}

Many thanks to all of the staff of the respiratory departments involved. Particular thanks to Alyson Pickup at Royal Blackburn Infirmary for her continued input and support.

\section{Funding source}

None.

\section{Authors' contributions}

$\mathrm{ML}$ conceived of the study, and participated in its design and coordination along with CS, RB, CSB, GB and LPO. CSB, MF, RM, RB, GB, HJM, NP and LPO collected the data. CSB collated and analysed the data with assistance from CS. CSB helped draft the manuscript with ML and CS. All authors contributed to and approved the final manuscript.

\section{Competing interests}

The authors declare that they have no competing interests.

\section{Author details}

${ }^{1}$ Hospital for Tropical Diseases, University College London Hospitals Foundation Trust, 235 Euston Road, London NW1 2BU, UK. ${ }^{2}$ UCL Division of Infection and Immunity, University College London, Rowland Hill Street, London NW3 2PF, UK. ${ }^{3}$ Royal Free Campus, University College London, Rowland Hill Street, London NW3 2PF, UK. ${ }^{4}$ Guy's and St Thomas' Hospital NHS Trust, Westminster Bridge Road, London SE1 7EH, UK. ${ }^{5}$ Royal Blackburn Hospital, Blackburn, Lancs BB2 3LR, UK. ' Royal Free London NHS Foundation Trust, Pond Street, London NW3 2QG, UK. ' Homerton University Hospital, Homerton Row, London E9 6SR, UK. ${ }^{8}$ UCL Respiratory, Division of Medicine, University College London, Rowland Hill Street, London NW3 2PF, UK.

Received: 27 April 2016 Accepted: 3 September 2016

Published online: 06 September 2016

References

1. Breen R, Smith C, Bettinson H, Dart S, Bannister B, Johnson M, Lipman M. Paradoxical reactions during tuberculosis treatment in patients with and without HIV co-infection. Thorax. 2004;59(8):704-7.

2. Lipman M, Breen R. Immune reconstitution inflammatory syndrome in HIV. Curr Opin Infect Dis. 2006;19(1):20-5.

3. Narita M, Ashkin D, Hollender E, Pitchenik A. Paradoxical worsening of tuberculosis following antiretroviral therapy inpatients with AIDS. Am J Respir Crit Care Med. 1998;158(1):157-61.

4. Meintjes G, Rabie H, Wilkinson RJ, Cotton MF. Tuberculosis-associated immune reconstitution inflammatory syndrome and unmasking of tuberculosis by antiretroviral therapy. Clin Chest Med. 2009:30(4):797-810.

5. Bell LC, Breen R, Miller RF, Noursadeghi M, Lipman M. Paradoxical reactions and immune reconstitution inflammatory syndrome in tuberculosis. Int J Infect Dis. 2015;32:39-45.

6. Coussens AK, Wilkinson RJ, Nikolayevskyy V, et al. Ethnic variation in inflammatory profile in tuberculosis. PLoS Pathog. 9(7): e1003468. doi:10.1371/journal.ppat.1003468.

7. Tostmann A, Boeree MJ, Aarnoutse RE, de Lange WC, van der Ven AJ, Dekhuijzen R. Antituberculosis drug-induced hepatotoxicity: concise up-todate review. J Gastroenterol Hepatol. 2008;23(2):192-202.

8. Ravimohan S, Tamuhla N, Steenhoff AP, et al. Immunological profiling of tuberculosis-associated immune reconstitution inflammatory syndrome and non-immune reconstitution inflammatory syndrome death in HIV-infected adults with pulmonary tuberculosis starting antiretroviral therapy: a prospective observational cohort study. Lancet Infect Dis. 2015;15(4):429-38. 
9. Chakrabarti LA, Boucherie C, Bugault F, et al. Biomarkers of CD4+ T-cell activation as risk factors for tuberculosis-associated immune reconstitution inflammatory syndrome. AIDS. 2014;28(11):1593-602.

10. Walker NF, Scriven J, Meintjes G, Wilkinson RJ. Immune reconstitution inflammatory syndrome in HIV-infected patients. HIV AIDS. 2015;7:49-64.

11. Cheng SL, Wang HC, Yang PC. Paradoxical response during anti-tuberculosis treatment in HIV-negative patients with pulmonary tuberculosis. Int J Tuberc Lung Dis. 2007;11(12):1290-5.

12. Cheng VC, Yam WC, Woo PC, Lau SK, Hung IF, Wong SP, Cheung WC, Yuen KY. Risk factors for development of paradoxical response during antituberculosis therapy in HIV-negative patients. Eur J Clin Microbiol Infect Dis. 2003;22(10):597-602.

13. Jung JW, Shin JW, Kim JY, Park IW, Choi BW, Seo JS, Choi JC. Risk factors for development of paradoxical response during anti-tuberculosis treatment in HIV-negative patients with pleural tuberculosis. Tohoku J Exp Med. 2011;223(3):199-204

14. Jeon K, Choi WI, An JS, et al. Paradoxical response in HIV-negative patients with pleural tuberculosis: a retrospective multicentre study. Int J Tuberc Lung Dis. 2012;16(6):846-51.

15. Brown CS, Breen R, Smith C, Bothamley G, Price N, Lilburn H, Lipman M. Ethnic determinants of paradoxical reaction during antituberculosis therapy. Poster Presentation A1685. New York: American Thoracic Society; 2009.

16. von Elm E, Altman DG, Egger M, et al. Strengthening the Reporting of Observational Studies in Epidemiology (STROBE) statement: guidelines for reporting observational studies. BMJ. 2007;335(7624):806-8.

17. Office for National Statistics. Ethnic Group. Webpage - http://ons.gov.uk/ ons/guide-method/measuring-equality/equality/ethnic-nat-identity-religion/ ethnic-group/index.html. Accessed 05 Nov 2015.

18. Lowe D. The ESR, in HIV: A Neglected Parameter? AIDS. 2010;42(18):2773-5.

19. Muller M, Wandel S, Colebunders R, Attia S, Furrer H, Egger M. Immune reconstitution inflammatory syndrome in patients starting antiretroviral therapy for HIV infection: a systematic review and meta-analysis. Lancet Infect Dis. 2010;10:251-61.

20. Park KH, Cho OH, Chong YP, et al. Post-therapy paradoxical response in immunocompetent patients with lymph node tuberculosis. J Infect. 2010; 61(5):430-4.

21. Asghar RJ, Pratt RH, Kammerer JS, Navin TR. Tuberculosis in South Asians living in the United States, 1993-2004. Arch Intern Med. 2008:168:936-42.

22. De D, Nath Sarkar R, Phaujdar S, Bhattacharyya K, Krishna PH. Incidence and risk factors of immune reconstitution inflammatory syndrome in HIV-TB coinfected patients. Braz J Infect Dis. 2011;15(6):553-9.

23. British Thoracic Society Research Committee. Six-months versus ninemonths chemotherapy for tuberculosis of lymph nodes: preliminary results. Respir Med. 1992;86:15-9.

24. Campbell IA, Ormerod LP, Friend JAR, Jenkins PA, Prescott RJ. Six-months versus nine-months chemotherapy for tuberculosis of lymph nodes: final results. Respir Med. 1993;87:621-3.

25. Meintjes G, Lawn SD, Scano F, et al. Tuberculosis-associated immune reconstitution inflammatory syndrome: case definitions for use in resource-limited settings. Lancet Infect Dis. 2008 Aug;8(8):516-23.

26. Marais S, Meintjes G, Pepper DJ, et al. Frequency, severity, and prediction of tuberculous meningitis immune reconstitution inflammatory syndrome. Clin Infect Dis. 2013;56(3):450-60.

27. Tan HY, Yong YK, Lim SH, et al. Tuberculosis (TB)-associated immune reconstitution inflammatory syndrome in TB-HIV co-infected patients in Malaysia: prevalence, risk factors, and treatment outcomes. Sex Health. 2014;11(6):532-9.

28. Alawin IA, Karnath BM. Paradoxical Immune Reconstitution Syndrome Presenting as Acute Respiratory Distress Syndrome in a Leukemia Patient during Neutrophil Recovery. Case Rep Hematol. 2012;2012:670347.

29. Belknap R, Reves R, Burman W. Immune reconstitution to Mycobacterium tuberculosis after discontinuing infliximab. Int J Tuberc Lung Dis. 2005;9(9):1057-8.

30. Haddow LJ, Dibben O, Moosa MY, Borrow P, Easterbrook PJ. Circulating inflammatory biomarkers can predict and characterize tuberculosis-associated immune reconstitution inflammatory syndrome. AIDS. 2011;25:1163-74.

31. Narendran G, Andrade BB, Porter BO, et al. Paradoxical Tuberculosis Immune Reconstitution Inflammatory Syndrome (TB-IRIS) in HIV Patients with Culture Confirmed Pulmonary Tuberculosis in India and the Potential Role of IL-6 in Prediction. PLoS One. 2013;8(5):e63541.

32. Jha BC, Dass A, Gupta R, Singhal S. Cervical tuberculous lymphadenopathy: changing clinical pattern and concepts in management. Postgrad Med J. 2001;77:185-7.

\section{Submit your next manuscript to BioMed Central and we will help you at every step:}

- We accept pre-submission inquiries

- Our selector tool helps you to find the most relevant journal

- We provide round the clock customer support

- Convenient online submission

- Thorough peer review

- Inclusion in PubMed and all major indexing services

- Maximum visibility for your research

Submit your manuscript at www.biomedcentral.com/submit
Biomed Central 